\title{
Synthesis and Antileishmanial Activity of Some Functionalized Peptoids
}

\author{
Daniel Previdi, ${ }^{a}$ Stephanie Rodrigues, ${ }^{a}$ Mike G. Coelho, ${ }^{a}$ Ana Carolina B. B. Candido, ${ }^{b}$ \\ Lizandra G. Magalhães ${ }^{b}$ and Paulo M. Donate ${ }^{\circledR} * a$ \\ ${ }^{a}$ Departamento de Química, Faculdade de Filosofia, Ciências e Letras de Ribeirão Preto, \\ Universidade de São Paulo, Avenida Bandeirantes 3900, 14040-901 Ribeirão Preto-SP, Brazil \\ ${ }^{b}$ Núcleo de Pesquisa em Ciências Exatas e Tecnológicas, Universidade de Franca, \\ Avenida Dr. Armando Salles de Oliveira 201, Parque Universitário, 14404-600 Franca-SP, Brazil
}

\begin{abstract}
We describe the microwave-assisted synthesis of thirteen functionalized peptoids and evaluate their in vitro antileishmanial activity against forms of Leishmania (Leishmania) amazonensis promastigotes. Synthesis via the Ugi four-component reaction (Ugi 4CR) reaction furnished the compounds of interest in 55-80\% yield; reactions were conducted in a microwave reactor and lasted only $10 \mathrm{~min}$. We then screened the antileishmanial activity of the synthesized compounds in vitro. To determine the $\mathrm{IC}_{50}$ (inhibitory concentration necessary to inhibit the growth of $50 \%$ of parasites) values, we selected the compounds that inhibited $L$. (L.) amazonensis growth by more than $50 \%$. The seven selected compounds displayed $\mathrm{IC}_{50}$ values ranging from 2.6 to $72 \mu \mathrm{M}$ after incubation for $48 \mathrm{~h}$. Three peptoids gave $\mathrm{IC}_{50}$ values between 2.6 and $7.9 \mu \mathrm{M}$ and can be considered as bioreactive molecules (hit criteria).
\end{abstract}

Keywords: peptoid, multicomponent reaction, Ugi reaction, microwave-assisted synthesis, Leishmania (Leishmania) amazonensis

\section{Introduction}

Protozoan parasites of the Leishmania genus cause a group of diseases known as leishmaniasis, a parasitosis that is transmitted by the bite of infected female phlebotomine sandflies in tropical and subtropical countries. ${ }^{1}$ These infections affect more than 12 million people, and an estimated 700,000 to $1,000,000$ new cases and 20,000 to 30,000 deaths occur annually. ${ }^{1-3}$ The infection manifests in three main typical ways: cutaneous leishmaniasis (LC), which presents skin lesions, mostly ulcers, on exposed body parts, leading to life-long scars and serious disability; mucocutaneous leishmaniasis (MCL), which partially or totally destroys mucous membranes; and visceral leishmaniasis (LV), which may be lethal if left untreated. ${ }^{1-3}$ Leishmania (Leishmania) amazonensis underlies a diffuse cutaneous form that may also result in visceral leishmaniasis in some cases in Latin America. ${ }^{4}$

Pentavalent antimonials were first used in the clinical setting at the beginning of the last century; they remain the first-choice drugs to treat leishmaniasis. However, these compounds are toxic and poorly tolerated, require daily

*e-mail: pmdonate@usp.br injections for up to 28 days, and are becoming ineffective due to proliferation of resistant parasites. ${ }^{5}$ Second-line drugs, like amphotericin B and pentamidine, are options in combined therapy or in cases of antimony treatment failure. ${ }^{6,7}$ Therefore, developing new antileishmanial compounds is imperative.

Peptoids are an emerging class of peptidomimetic molecules that offer an alternative to peptides. Peptoids consist of $\mathrm{N}$-substituted glycines where side-chains are located on the amide backbone nitrogen atom rather than the $\alpha$-carbon in the case of peptides (see Figure 1). ${ }^{8}$ This structural change makes the molecule highly resistant to proteolytic degradation, rendering peptoids a higher stability under physiological conditions and an improved biological lifetime. These non-natural molecules are being increasingly investigated because they are easy to synthesize, and various functionalities can be incorporated into their amide side-chains, which may generate new compounds with several biological activities and interesting pharmaceutical properties. ${ }^{9-12}$

The Ugi reaction is a multicomponent process that is widely applied in the pharmaceutical industry to prepare libraries of compounds. ${ }^{13}$ This reaction is very useful to produce peptoid-like backbones that occur not only in 


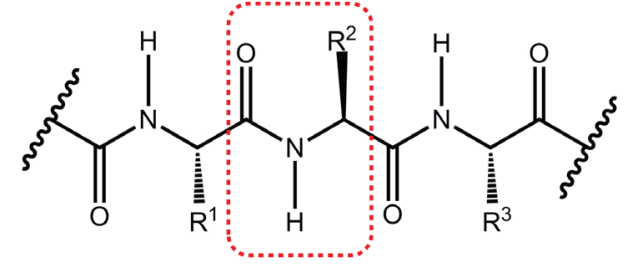

(a) peptide

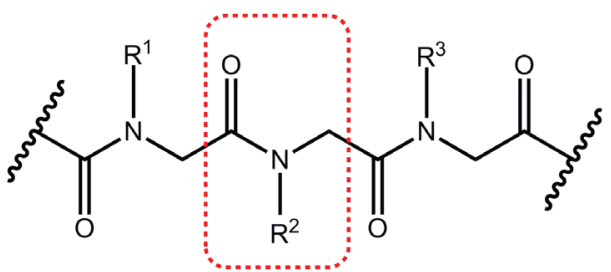

(b) peptoid

Figure 1. Representative structures of (a) peptide and (b) peptoid backbone.

peptides, but also in many other biologically important heterocycles. ${ }^{14}$ Besides its value in Medicinal Chemistry, the Ugi reaction has gained increased acceptance because it is easily performed in almost any solvent, and it is environmentally friendly. ${ }^{15}$

As part of an ongoing program to develop methods to synthesize biologically active compounds, ${ }^{16}$ in this study we examine how the classical Ugi reaction (Ugi four-component reaction (Ugi 4CR)) can be employed to synthesize a small library of new peptoids with potential pharmacological activity. ${ }^{17}$ Because some peptoids have been shown to display promising activity against Leishmania, we also evaluate the antileishmanial activity of these compounds. ${ }^{18}$

\section{Experimental}

\section{General}

High-resolution mass spectrometry (HRMS) were recorded on a Bruker Daltonics micrOTOF II-ESI-TOF (Billerica, MA, USA) operating in the positive ion mode. The samples were diluted in acetonitrile and water $(1: 1$, $\mathrm{v} / \mathrm{v}$ ) at a concentration of $0.02 \mathrm{mg} \mathrm{mL}^{-1}$. Accurate mass measurements were achieved using sodiated trifluoroacetic acid (TFA- $\mathrm{Na}^{+}$) as standard for internal calibration on electrospray ionization time of flight (ESI-TOF). Molecular formulas were assigned from accurate mass and exact mass errors lower than $5 \mathrm{ppm} .{ }^{1} \mathrm{H}$ nuclear magnetic resonance (NMR) spectroscopy was performed on a Bruker DPRX 400 instrument (Bruker, Fällanden, Switzerland) operating at $400 \mathrm{MHz}$ for ${ }^{1} \mathrm{H}$ and at $100 \mathrm{MHz}$ for ${ }^{13} \mathrm{C}$. Tetramethylsilane (TMS) was used as internal standard. Chemical shifts are reported in ppm $(\delta)$; coupling constants $(J)$ are given in hertz $(\mathrm{Hz})$. Signal multiplicities are represented by: s (singlet), d (doublet), dd (double doublet), dq (double quadruplet), and $\mathrm{m}$ (multiplet). Infrared (IR) spectra were recorded on a PerkinElmer Spectrum RX IFTIR System (Waltham, MA, USA), in $\mathrm{KBr}$ pellets or plates. Unless noted otherwise, all solvents and reagents were commercially available and used without further purification.

\section{General procedure to synthesize peptoids $5 \mathbf{a}-\mathbf{m}$}

Preparation of peptoids $\mathbf{5 a - m}$ by the Ugi four-component reaction (Ugi 4CR)

Peptoids 5a-m were synthesized via one-pot multicomponent methodology; the Ugi four-component reaction (Ugi 4CR) was employed. ${ }^{13-15}$ Reactions were conducted with the corresponding aldehyde $1(2 \mathrm{mmol})$, amine $\mathbf{2}$ ( $2 \mathrm{mmol})$, isocyanide $\mathbf{3}(1 \mathrm{mmol})$, and carboxylic acid 4 (2 mmol) in a 5-mL round bottom flask under argon atmosphere. ${ }^{10}$ Anhydrous $\mathrm{MgSO}_{4}(0.05 \mathrm{~g})$ was added to the flask, and the resulting suspension was heated at $60^{\circ} \mathrm{C}$ for $10 \mathrm{~min}$ in a CEM Discovery ${ }^{\circledR}$ focused microwave oven at $150 \mathrm{~W}$. After that, the reaction mixture was filtered, the solid was washed several times with anhydrous methanol, and this solvent was removed under reduced pressure. The residue was purified by column chromatography on silica gel with dichloromethane/methanol (95:5, v/v) as eluent, to give the peptoids 5a-m (55-80\% yields).

\section{In vitro antileishmanial assays}

The antileishmanial activity was assayed with L. (L.) amazonensis promastigotes (MHOM/BR/PH8), which were maintained in culture medium Roswell Park Memorial Institute (RPMI) 1640 (Cultilab, Campinas, SP, Brazil) supplemented with $10 \%$ fetal bovine serum (Cultilab, Campinas, SP, Brazil), and $1 \%$ antibiotics (10,000 UI mL $\mathrm{mL}^{-1}$ penicillin and $10 \mathrm{mg} \mathrm{mL}^{-1}$ streptomycin) (Cultilab, Campinas, SP, Brazil), at pH 7.4 at $25^{\circ} \mathrm{C}$.

Five days after the culture was initiated, the antileishmanial activity was screened in 96-well microplates containing $1 \times 10^{6}$ L. (L.) amazonensis promastigotes in supplemented RPMI 1640 and $100 \mu \mathrm{M}$ of the compounds previously dissolved in dimethyl sulfoxide (DMSO) (Synth, Diadema, SP, Brazil). Cultures were incubated in biological oxygen demand (BOD) incubator (Quimis, Diadema, SP, Brazil) at $25^{\circ} \mathrm{C}$ for 24 and $48 \mathrm{~h}$, and the antileishmanial activity was determined by verifying whether the promastigote growth was inhibited, as revealed by counting the total number of live promastigotes in the Neubauer chamber (Global Glass, Porto Alegre, RS, Brazil), on the basis of flagellar motility. ${ }^{19}$ 
The peptoids that inhibited $L$. (L.) amazonensis growth by at least $50 \%$ in $48 \mathrm{~h}$ were evaluated at final concentrations of $6.25,12.5,25.0,50.0$, and $100.0 \mu \mathrm{M}$. Bioassays were repeated three times, in triplicate. Amphotericin B (at concentration ranging from 0.6 to $0.038 \mu \mathrm{M}$ or $1 \mu \mathrm{M}$ ) (Sigma-Aldrich, Saint Louis, MO, USA) was used as positive control, and RPMI 1640 medium with $0.1 \%$ DMSO was employed as negative control. Results are expressed as the mean of the percentage of growth inhibition relative to the negative control $\left(0.1 \%\right.$ DMSO), and the $\mathrm{IC}_{50}$ (inhibitory concentration necessary to inhibit the growth of $50 \%$ of parasites) values were calculated by using sigmoid doseresponse curves constructed with the GraphPad Prism version 5.0 software for Windows. ${ }^{20}$

\section{Results and Discussion}

Microwave irradiation is a relevant tool for fast and efficient synthesis of compound libraries. Microwaveassisted reactions are advantageous over reactions conducted by conventional heating because they take shorter reaction times and generate fewer by-products..$^{21,22}$

We obtained the target compounds by one-pot Ugi 4CR from less expensive raw materials (see Scheme 1), by using microwave irradiation as heat source. ${ }^{10}$ Table 1 summarizes the results of these reactions.

We confirmed the structures of all the synthesized compounds by ${ }^{1} \mathrm{H}$ and ${ }^{13} \mathrm{C}$ NMR, Fourier transform IR (FTIR), and high-resolution mass spectral data.

In the biological assays, we tested all the compounds as mixtures of enantiomers or diastereoisomers. Table 2 lists the results of the in vitro antileishmanial evaluation of peptoids 5a-m against Leishmania (L.) amazonensis promastigotes (MHOM/BR/PH8).

First, we screened the antileishmanial activity of the synthesized compounds at $100 \mu \mathrm{M}$ for 24 and $48 \mathrm{~h}$, in vitro, as shown in Table 2. Among the evaluated compounds, peptoids $\mathbf{5 b}, \mathbf{5 c}$, and $5 \mathbf{e}$ inhibited $L$. (L.) amazonensis promastigote growth by approximately $50 \%$ within
48 h. Peptoids $5 \mathbf{5 a}, \mathbf{5 d}, \mathbf{5 f}, \mathbf{5 g}, \mathbf{5 j}$, and $\mathbf{5 l}$ inhibited L. (L.) amazonensis promastigote growth by more than $50 \%$ within $48 \mathrm{~h}$ (see entries 1, 3, 4, 6, 7, 10 and 12 in Table 2). Peptoid 5j provided the most promising result: 66.83 and $87.87 \%$ L. (L.) amazonensis promastigote growth inhibition within 24 and $48 \mathrm{~h}$, respectively. We then selected seven of the tested peptoids to perform future biological studies.

We assessed the seven selected peptoids $(\mathbf{5 a}, \mathbf{5 c}, \mathbf{5 d}, \mathbf{5 f}$, $\mathbf{5 g}, \mathbf{5 j}$, and $\mathbf{5 l}$ ) at $6.25,12.5,25,50$, and $100 \mu \mathrm{M}$ in vitro for $48 \mathrm{~h}$ against $L$. ( $L$.) amazonensis promastigotes to determine the $\mathrm{IC}_{50}$ values (Table 3 ). These seven compounds displayed $\mathrm{IC}_{50}$ values ranging from 2.6 to $72 \mu \mathrm{M}$ after incubation for $48 \mathrm{~h}$ (Table 3 ). We used amphotericin $\mathrm{B},{ }^{23}$ a polyene antibiotic that binds to specific parasite sterols, as positive control and achieved an $\mathrm{IC}_{50}$ value of $0.11 \mu \mathrm{M}$ at $48 \mathrm{~h}$.

When we compared the percentage of $L$. (L.) amazonensis promastigote growth inhibition by the peptoids 5a-m (Table 2), we observed that the antileishmanial activity decreased when a benzenoid aromatic ring was replaced by a furan ring, as in the case of compounds $\mathbf{5 a}$ and $\mathbf{5 k}$. The same occurred when the peptoid acetamido group was replaced by a long chain $n$-alkylamido group, as in the case of compound $\mathbf{5 m}$. When we related the $\mathrm{IC}_{50}$ values to the peptoid structure (Table 3), we verified that the antileishmanial activity was higher for benzamides than for acetamides, as in the case of compounds $\mathbf{5 a}$ and $\mathbf{5 d}$. However, the presence of an $\mathrm{N}$-sec-butylacetamido group greatly increased the antileishmanial activity in relation to the $N$-n-butylacetamido group, as in the case of compounds $\mathbf{5 a}$ and $\mathbf{5 c}$. On the other hand, the presence of an $\mathrm{N}$-(1-phenylethyl)acetamido group significantly decreased the antileishmanial activity, as in the case of compounds $\mathbf{5 f}$ and $\mathbf{5 g}$. Apparently, aromatic rings in the peptoid side chain increase the antileishmanial activity if we compare the activities of compounds $\mathbf{5 c}, \mathbf{5 e}, \mathbf{5 i}, \mathbf{5 j}$, and 5l. Despite all these considerations, further studies are necessary to investigate how these substituents affect the antileishmanial activity of this class of compounds.

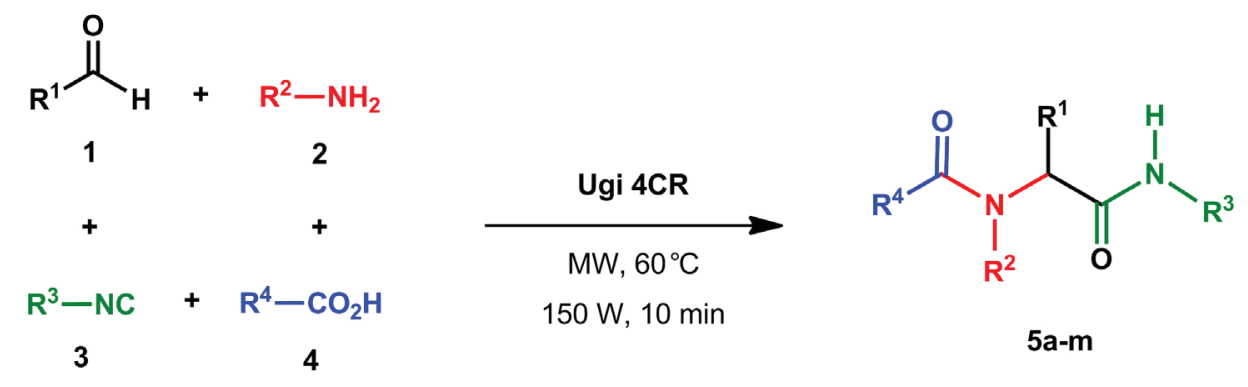

$\left(\mathbf{R}^{1}, \mathbf{R}^{2}, \mathbf{R}^{3}, \mathbf{R}^{4}=\right.$ alkyl or aryl)

Scheme 1. Preparation of compounds $\mathbf{5 a}-\mathbf{m}$ by the Ugi four-component reaction (Ugi 4CR). 
Table 1. Yields obtained in the synthesis of compounds $\mathbf{5 a}-\mathbf{m}$ produced by Ugi $4 \mathrm{CR}$ via Scheme $1^{\text {a }}$

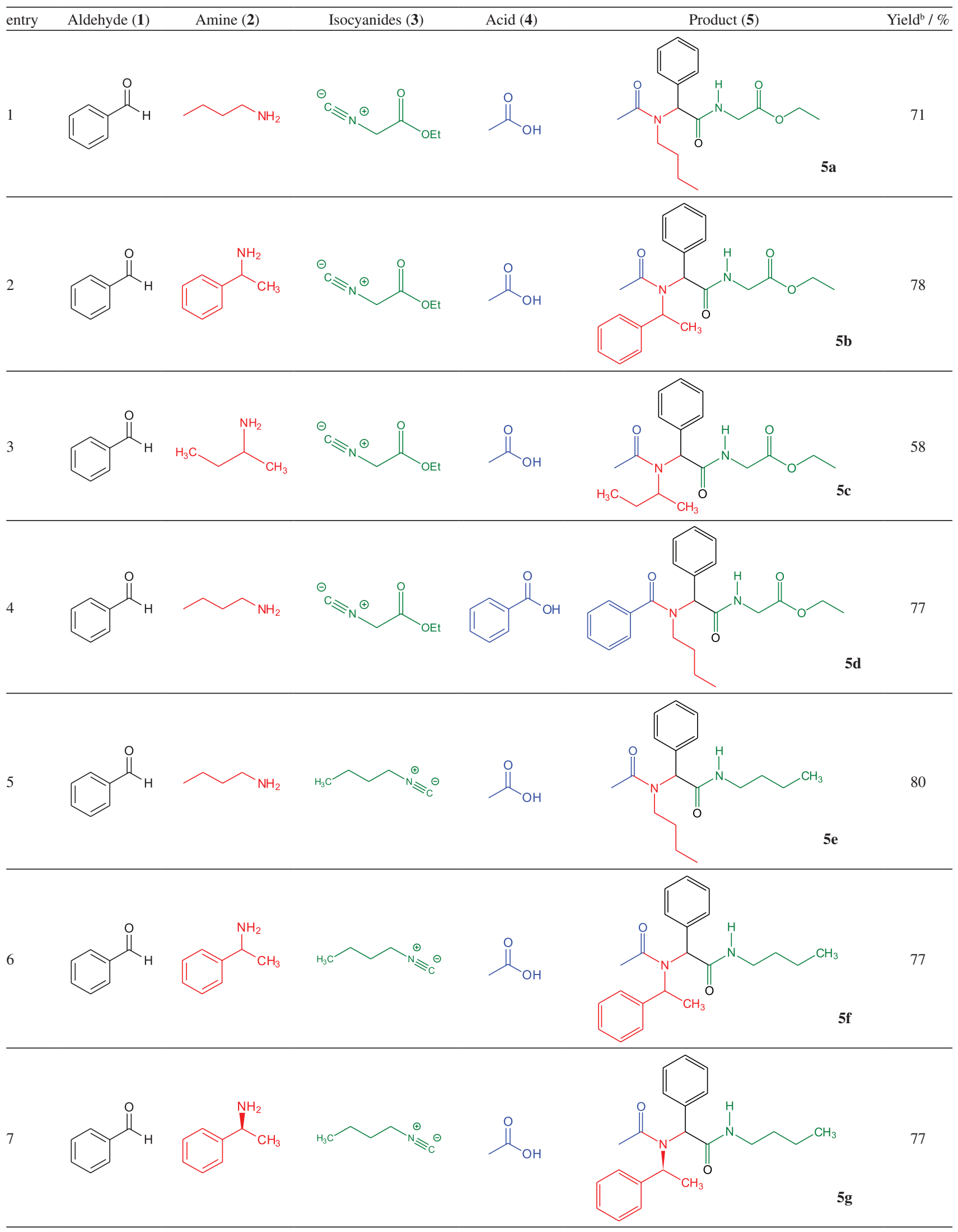


Table 1. Yields obtained in the synthesis of compounds $\mathbf{5 a - m}$ produced by Ugi $4 \mathrm{CR}$ via Scheme $1^{\mathrm{a}}$ (cont.)<smiles>C#[N+]CCN1CCOCC1</smiles>

10<smiles>CCCCN</smiles><smiles>Cc1ccc(S(=O)(=O)C[N+](=O)[O-])cc1</smiles><smiles>CC(=O)O</smiles><smiles>CCN(C(C)=O)C(C(=O)NCS(=O)(=O)c1ccc(C)cc1)c1ccccc1</smiles><smiles>O=Cc1ccccc1</smiles>

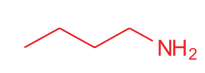<smiles>O=[N+]=[N+]=[N+]([O-])Cn1nnc2ccccc21</smiles><smiles>CC(=O)O</smiles><smiles>CCN(C(C)=O)C(C(=O)NCn1nnc2ccccc21)c1ccccc1</smiles>

51<smiles>CCCCN</smiles><smiles>CCOC(=O)CN=[O+]</smiles><smiles>CCCCCCC(C)=O</smiles><smiles>COC(=O)OC</smiles><smiles>c1ccccc1</smiles><smiles>C=C(C)C(NCCCC)C(=O)CC</smiles><smiles>C1CCN2CCCN2C1</smiles><smiles>CCCCCOC(C)=O</smiles>

${ }^{a}$ Reactions were conducted with the corresponding aldehyde $\mathbf{1}(2 \mathrm{mmol})$, amine $\mathbf{2}(2 \mathrm{mmol})$, isocyanide $\mathbf{3}(1 \mathrm{mmol})$ and carboxylic acid $\mathbf{4}(2 \mathrm{mmol})$ in a 5 -mL round bottom flask under argon atmosphere. Anhydrous $\mathrm{MgSO}_{4}(0.05 \mathrm{~g})$ was added to the flask, and the resulting suspension was heated at $60{ }^{\circ} \mathrm{C}$ for $10 \mathrm{~min}$ in a CEM Discovery ${ }^{\circledR}$ focused microwave oven at $150 \mathrm{~W}$. After that, the reaction mixture was filtered, the solid was washed several times with anhydrous methanol, and this solvent was removed under reduced pressure. The residue was purified by column chromatography on silica gel with dichloromethane/methanol (95:5, v/v) as eluent, to give peptoids 5a-m (55-80\% yields). All the products were analyzed by NMR, FTIR, and high-resolution mass spectral data. The obtained spectra were consistent with the structures of the desired products; ${ }^{b}$ isolated yield. 
Table 2. In vitro antileishmanial activity of peptoids $\mathbf{5 a}-\mathbf{m}$ at $100 \mu \mathrm{M}$ against $L$. (L.) amazonensis promastigotes

\begin{tabular}{lccc}
\hline entry & Compound & $\begin{array}{c}\text { Inhibition } \pm \text { SD } \\
(24 \mathrm{~h}) / \%\end{array}$ & $\begin{array}{c}\text { Inhibition } \pm \text { SD } \\
(48 \mathrm{~h}) / \%\end{array}$ \\
\hline 1 & $\mathbf{5 a}$ & $11.84 \pm 9.97$ & $54.64 \pm 9.21$ \\
2 & $\mathbf{5 b}$ & $29.37 \pm 5.3$ & $45.80 \pm 0.66$ \\
3 & $\mathbf{5 c}$ & $28.24 \pm 2.68$ & $81.33 \pm 1.52$ \\
4 & $\mathbf{5 d}$ & $31.91 \pm 6.96$ & $81.94 \pm 15.81$ \\
5 & $\mathbf{5 e}$ & $26.95 \pm 11.42$ & $45.06 \pm 3.54$ \\
6 & $\mathbf{5 f}$ & $25.98 \pm 11.31$ & $67.41 \pm 9.01$ \\
7 & $\mathbf{5 g}$ & $20.11 \pm 1.08$ & $55.21 \pm 0.51$ \\
8 & $\mathbf{5 h}$ & $12.01 \pm 5.41$ & $13.22 \pm 5.97$ \\
9 & $\mathbf{5 i}$ & $0 \pm 0$ & $1.64 \pm 2.32$ \\
10 & $\mathbf{5 j}$ & $66.83 \pm 12.19$ & $87.87 \pm 21.36$ \\
11 & $\mathbf{5 k}$ & $0 \pm 0$ & $25.14 \pm 1.06$ \\
12 & $\mathbf{5 l}$ & $5.32 \pm 2.40$ & $51.27 \pm 9.95$ \\
13 & $\mathbf{5 m}$ & $8.72 \pm 3.80$ & $24.05 \pm 10.83$ \\
14 & amphotericin B & $100 \pm 0.0$ & $100 \pm 0.0$ \\
\hline Positive control: amphotericin B $(1 \mu \mathrm{M})$. SD: standard deviation.
\end{tabular}

Positive control: amphotericin B (1 $\mu \mathrm{M})$. SD: standard deviation.

According to hit-and-lead criteria, ${ }^{24}$ the hit is a bioreactive compound, and the lead is a hit compound that has been optimized and has undergone improvements in its pharmacokinetic and pharmacodynamic properties, for example. In addition, for a compound to be considered a hit it must have $\mathrm{IC}_{50}<10 \mu \mathrm{M}$ against protozoan parasites, which is the inhibitory concentration reached by peptides $\mathbf{5 c}$ $(2.8 \mu \mathrm{M}), \mathbf{5 d}(2.6 \mu \mathrm{M})$, and $\mathbf{5 j}(7.9 \mu \mathrm{M})$. Therefore, these three peptoids have potent in vitro activity against L. (L.) amazonensis, making our results very promising. Furthermore, several peptoids have recently been shown to act against Leishmania (Leishmania) mexicana promastigotes with $\mathrm{IC}_{50}$ values of $\leq 20 \mu \mathrm{M} .{ }^{18}$ These results provide further evidence that peptoids may be a promising new class of anti-infectives and will surely find application as a new class of antileishmanial agents. ${ }^{25}$

\section{Conclusions}

In summary, we have presented an easy and efficient method to obtain some functionalized peptoids by using microwave irradiation as the activating mode of reaction. We conducted the syntheses with inexpensive reagents within shorter reaction times as compared to traditional methods. This method, microwave-assisted synthesis by Ugi 4CR, furnished functionalized peptoids (compounds 5a-m) in good yields (55-80\%). All the compounds were evaluated against Leishmania (Leishmania) amazonensis promastigotes in vitro. Peptoids 5a-m displayed variable antileishmanial activity depending on their structures. On the basis of our results, peptoids $\mathbf{5 c}, \mathbf{5 d}$, and $\mathbf{5 j}$ display the best antileishmanial activity at the assayed concentrations $\left(\mathrm{IC}_{50}\right.$ 2.6-7.9 $\left.\mu \mathrm{M}\right)$. Now, it is necessary to gain further understanding about their structure-biological activity relationships to expand the application of this kind of peptoids.

\section{Supplementary Information}

${ }^{1} \mathrm{H}$ NMR, ${ }^{13} \mathrm{C}$ NMR, FTIR and high-resolution mass spectra of compounds, and the curves used to calculate the $\mathrm{IC}_{50}$ values are available free of charge at http://jbcs.sbq.org.br as PDF file.

\section{Acknowledgments}

The authors would like to thank FAPESP (grant

Table 3. In vitro antileishmanial activity of $L$. (L.) amazonensis promastigotes and determination of $\mathrm{IC}_{50}$ values after incubation for $48 \mathrm{~h}$

\begin{tabular}{|c|c|c|c|c|c|c|}
\hline \multirow{3}{*}{ Compound } & \multicolumn{5}{|c|}{ Concentration \pm standard deviation $/ \mu \mathrm{M}$} & \multirow{3}{*}{$\mathrm{IC}_{50}{ }^{\mathrm{a}} / \mu \mathrm{M}$} \\
\hline & \multicolumn{5}{|c|}{ Parasite growth inhibition / \% } & \\
\hline & 100 & 50 & 25 & 12.5 & 6.25 & \\
\hline $5 \mathbf{a}$ & $56.27 \pm 0.57$ & $45.86 \pm 5.76$ & $42.05 \pm 5.61$ & $34.78 \pm 7.55$ & $29.11 \pm 7.02$ & $60.60 \pm 0.39$ \\
\hline $5 c$ & $81.42 \pm 5.50$ & $74.12 \pm 2.28$ & $68.17 \pm 2.85$ & $63.83 \pm 4.30$ & $58.47 \pm 8.11$ & $2.80 \pm 0.38$ \\
\hline 5d & $84.23 \pm 11.59$ & $78.12 \pm 13.16$ & $69.75 \pm 6.26$ & $64.60 \pm 4.94$ & $61.01 \pm 2.97$ & $2.61 \pm 0.42$ \\
\hline $5 f$ & $58.61 \pm 5.27$ & $45.90 \pm 0.98$ & $38.37 \pm 2.06$ & $30.12 \pm 4.53$ & $23.79 \pm 3.66$ & $59.03 \pm 0.54$ \\
\hline $5 g$ & $52.88 \pm 1.59$ & $49.30 \pm 0.99$ & $34.48 \pm 0.74$ & $28.84 \pm 1.64$ & $25.25 \pm 1.06$ & $72.00 \pm 0.48$ \\
\hline $5 \mathbf{j}$ & $75.92 \pm 2.97$ & $67.59 \pm 0.52$ & $62.61 \pm 0.33$ & $53.48 \pm 0.56$ & $48.54 \pm 1.12$ & $7.90 \pm 0.42$ \\
\hline $5 \mathbf{l}$ & $69.23 \pm 3.17$ & $63.66 \pm 2.44$ & $55.70 \pm 5.63$ & $48.20 \pm 7.50$ & $43.41 \pm 8.55$ & $13.35 \pm 0.40$ \\
\hline \multirow{2}{*}{ Amphotericin B } & 0.6 & 0.3 & 0.15 & 0.075 & 0.038 & \multirow{2}{*}{$0.11 \pm 0.34$} \\
\hline & $99.88 \pm 0.60$ & $78.33 \pm 24.43$ & $68.74 \pm 21.97$ & $54.67 \pm 17.11$ & $42.44 \pm 20.97$ & \\
\hline
\end{tabular}

Inhibition concentration $50\left(\mathrm{IC}_{50}\right)$ values were calculated by using a nonlinear regression curve. Negative control: RPMI 1640 medium + 0.1\% DMSO. 
No. 2015/05627-7, and 2016/04896-7), CAPES (Finance Code 001) and CNPq (grant No. 162749/2015-5) for financial support and fellowships.

\section{References}

1. http://www.who.int/news-room/fact-sheets/detail/leishmaniasis, accessed on August 8, 2018.

2. Okwor, I.; Uzonna, J.; Am. J. Trop. Med. Hyg. 2016, 94, 489.

3. Alvar, J.; Vélez, I. D.; Bern, C.; Herrero, M.; Desjeux, P.; Cano, J.; Jannin, J.; den Boer, M.; PLoS One 2012, 7, e35671.

4. Torres-Guerrero, E.; Quintanilla-Ceillo, M. R.; RuizEsmenjaud, J.; Arenas, R.; F1000Research 2017, 6, 750. DOI: 10.12688/f1000research.11120.1.

5. Monzote, L.; Garcia, M.; Montalvo, A. M.; Scull, R.; Miranda, M.; Mem. Inst. Oswaldo Cruz 2010, 105, 168.

6. Monzote, L.; Montalvo, A. M.; Almanonni, S.; Scull, R.; Miranda, M.; Abreu, J.; Barral, A.; Chemotherapy 2006, 52, 130.

7. Santin, M. R.; Santos, A. O.; Nakamura, C. V.; Dias Filho, B. P.; Ferreira, I. C. P.; Ueda-Nakamura, T.; Parasitol. Res. 2009, $105,1489$.

8. Fowler, S. A.; Blackwell, H. E.; Org. Biomol. Chem. 2009, 7, 1508; Zuckermann, R. N.; Biopolymers 2011, 96, 545; Sun, J.; Zuckermann, R. N.; ACS Nano 2013, 7, 4715.

9. Seo, J.; Barron, A. E.; Zuckermann, R. N.; Org. Lett. 2010, 12, 492.

10. Barreto, A. F. S.; Vercillo, O. E.; Birkett, M. A.; Caulfield, J. C.; Wessjohann, L.; Andrade, C. K. Z.; Org. Biomol. Chem. 2011, 9, 5024.

11. Barreto, A. F. S.; Vercillo, O. E.; Wessjohann, L.; Andrade, C. K. Z.; Beilstein J. Org. Chem. 2014, 10, 1017.

12. Barreto, A. F. S.; Santos, V. A.; Andrade, C. K. Z.; Beilstein J. Org. Chem. 2016, 12, 2865.

13. Zhu, J.; Wang, Q.; Multicomponent Reactions in Organic Synthesis; Wiley-VCH: Weinheim, Germany, 2014; Dömling, A.; Wang, W.; Wang, K.; Chem. Rev. 2012, 112, 3083.
14. Dömling, A.; Chem. Rev. 2006, 106, 17.

15. Huang, Y.; Yazbak, A.; Dömling, A. In Green Techniques for Organic Synthesis and Medicinal Chemistry; Zhang, W.; Cue Jr., B. W., eds.; Wiley: Chichester, UK, 2012, p. 497-522.

16. Silva, E. H. B.; Emery, F. S.; Del Ponte, G.; Donate, P. M.; Synth. Commun. 2015, 45, 1761.

17. Dohm, M. T.; Kapoor, R.; Barron, A. E.; Curr. Pharm. Des. 2011, 17, 2732.

18. Eggimann, G. A.; Bolt, H. L.; Denny, P. W.; Cobb, S. L.; ChemMedChem 2015, 10, 233; Bolt, H. L.; Eggimann, G. A.; Denny, P. W.; Cobb, S. L.; Med. Chem. Commun. 2016, 7, 799; Bolt, H. L.; Denny, P. W.; Cobb, S. L.; J. Visualized Exp. 2016, 117, e54750.

19. Azzouz, S.; Maache, M.; Garcia, R. G.; Osuna, A.; Basic Clin. Pharmacol. Toxicol. 2005, 96, 60.

20. GraphPad Prism, version 5.0 software for Windows; GraphPad Software Inc., San Diego, USA, 2007.

21. Kappe, C. O.; Stadler, A.; Microwaves in Organic and Medicinal Chemistry; Wiley-VCH: Verlag, Weinheim, Germany, 2005; Loupy, A.; Microwaves in Organic Synthesis, $2^{\text {nd }}$ ed.; WileyVCH: Weinheim, Germany, 2006.

22. Surati, M. A.; Jauhari, S.; Desai, K. R.; Arch. Appl. Sci. Res. 2012, 4, 645; Kappe, C. O.; Pieber, B.; Dallinger, D.; Angew. Chem., Int. Ed. 2013, 52, 1088.

23. Machado, P. R.; Rosa, M. E.; Guimarães, L. F.; Prates, F. V.; Queiroz, A.; Schriefer, A.; Carvalho, E. M.; Clin. Infect. Dis. 2015, 61, 945 .

24. Katsuno, K.; Burrows, J. N.; Duncan, K.; van Huijsduijnen, R. H.; Kaneko, T.; Kita, K.; Mowbray, C. E.; Schmatz, D.; Warner, P.; Slingsby, B. T.; Nat. Rev. Drug Discovery 2015, 14, 751.

25. Zulfiqar, B.; Shelper, T. B.; Avery, V. M.; Drug Discovery Today 2017, 22, 1516.
Submitted: October 22, 2018

Published online: February 7, 2019 